\title{
ON THE ERROR IN A CERTAIN INTERPOLATION FORMULA AND IN THE GAUSSIAN INTEGRATION FORMULA
}

\author{
YUDELL L. LUKE \\ (Received 9 May 1972; revised 15 August 1972) \\ Communicated by B. Mond
}

\begin{abstract}
We first prove a basic theorem that if a set of polynomials satisfies an orthogonality relation with respect to integration, the set also satisfies an orthogonality relation with respect to summation. This result is then used to derive the Gaussian quadrature formula. The orthogonality relations give rise to interpolation formulas and a connection between the coefficients in these interpolation formulas is established. Finally, the analysis is used to get an estimate of the error in the Gaussian quadrature formula. Some error coefficients are evaluated in the cases where the orthogonal polynomials are those of Jacobi, Laguerre, Hermite and Bessel.
\end{abstract}

\section{Introduction}

In this section, we set down some key relations needed to establish a basic theorem and some of its consequences. For the most part, we omit proofs as the results are well known and are given in a number of standard references. Let

$$
q_{n}(x)=\sum_{k=0}^{n} a_{k, n} x^{k}
$$

be a set of orthogonal polynomials over the interval $[a, b]$ with respect to the nonnegative weight function $w(x), w(x) \geqq 0$, so that

$$
\int_{a}^{b} w(x) q_{n}(x) q_{m}(x) d x=h_{n} \delta_{m n}
$$

where $\delta_{m n}$ is the usual notation for the Kronecker delta function. The polynomials $q_{n}(x)$ satisfy the three term recurrence relation

$$
q_{n+1}(x)=\left(A_{n} x+B_{n}\right) q_{n}(x)-C_{n} q_{n-1}(x), \quad n>0,
$$

This research was sponsored by the Air Force Office of Scientific Research. 


$$
q_{1}(x)=\left(A_{0} x+B_{0}\right) q_{0}(x)
$$

where

$$
\begin{array}{ll}
A_{n}=\frac{a_{n+1, n+1}}{a_{n, n}} ; & C_{n}=\frac{A_{n} h_{n}}{A_{n-1} h_{n-1}}, n>0 ; \\
B_{n}=A_{n}\left(r_{n+1}-r_{n}\right) ; & r_{n}=\frac{a_{n-1, n}}{a_{n, n}}, \quad n>0, r_{0}=0 .
\end{array}
$$

As a consequence of the recursion relation, we have the Christoffel-Darboux formula

$$
\sum_{k=0}^{n} h_{k}^{-1} q_{k}(x) q_{k}(y)=\left(A_{n} h_{n}\right)^{-1} \frac{q_{n+1}(x) q_{n}(y)-q_{n}(x) q_{n+1}(y)}{x-y} .
$$

We suppose throughout that $f(x)$ can be represented by an expansion in series of polynomials $\left\{q_{n}(x)\right\}$ which is uniformly convergent in $[a, b]$. Thus

$$
\begin{aligned}
f(x) & =\sum_{k=0}^{\infty} c_{k} q_{k}(x), \\
c_{k} & =h_{k}^{-1} \int_{a}^{b} w(x) q_{k}(x) f(x) d x .
\end{aligned}
$$

Suppose that $f_{n}(x)$ is an $n$th degree polynomial approximation to $f(x)$ such that

$$
f\left(x_{\alpha}\right)=f_{n}\left(x_{\alpha}\right), \alpha=0,1, \cdots, n, q_{n+1}\left(x_{\alpha}\right)=0 .
$$

Then by the Lagrangian interpolation formula, we have

$$
\begin{aligned}
& f(x)=f_{n}(x)+R_{n+1}(x), \\
& f_{n}(x)=\sum_{\alpha=0}^{n} \frac{q_{n+1}(x) f\left(x_{\alpha}\right)}{\left(x-x_{\alpha}\right) q_{n+1}^{\prime}\left(x_{\alpha}\right)},
\end{aligned}
$$

where $R_{n+1}(x)$ is the remainder. The remainder is usually expressed in terms of a certain divided difference or derivative of $f(x)$. If $f(x)$ is analytic, it can also be expressed as a Cauchy contour integral involving $f(x)$. We do not record these expressions as they can be found in various sources. For a complete discussion, see Davis (1963). Later, we derive an expression for the remainder in terms of the coefficients $c_{k}$.

In the Christoffel-Darboux formula (7), put $y=x_{\alpha}$ and combine with (12) to get

$$
f_{n}(x)=\sum_{k=0}^{n} d_{k, n} q_{k}(x)
$$




$$
d_{k, n}=\frac{A_{n} h_{n}}{h_{k}} \sum_{\alpha=0}^{n} \frac{f\left(x_{\alpha}\right) q_{k}\left(x_{\alpha}\right)}{q_{n+1}^{\prime}\left(x_{\alpha}\right) q_{n}\left(x_{\alpha}\right)}
$$

\section{A basic theorem}

We now show that if a set of polynomials satisfies an orthogonality relation with respect to integration, the set also satisfies an orthogonality relation with respect to summation. In particular we prove the following

THEOREM. Let

$$
V_{j, k}^{(n)}=\frac{A_{n} h_{n}}{h_{k}} \sum_{x=0}^{n} \frac{q_{j}\left(x_{\alpha}\right) q_{k}\left(x_{\alpha}\right)}{q_{n+1}^{\prime}\left(x_{x}\right) q_{n}\left(x_{x}\right)}, q_{n+1}\left(x_{x}\right)=0 .
$$

Then

$$
V_{j, k}^{(n)}=\delta_{j k}, \quad j \leqq n, k \leqq n .
$$

Proof. The representation (11) is exact if $f(x)$ is a polynomial of degree $\leqq n$. If $f(x)=q_{j}(x), f_{n}(x)=q_{j}(x)$ for $j \leqq n$ and for such an $f_{n}(x)$, it follows from (13) that $d_{k, n}=1$ if $k=j$ and $d_{k, n}=0$ if $k \neq j$. In (14), put $f(x)=q_{j}(x)$ and call the resulting expression $V_{j, k}^{(n)}$. We therefore have (15) and (16) and the theorem is proved.

\section{Corollary 1.}

$$
\begin{aligned}
& h_{k} V_{j, k}^{(n)}=h_{j} V_{k, j}^{(n)} \text { for all } j \text { and } k \text {, } \\
& V_{j, n+1}^{(n)}=V_{n+1, k}^{(n)}=0 \text { for all } j \text { and } k \text {. } \\
& V_{n+2, k}^{(n)}=-C_{n+1} V_{n+k}^{(n)} \text {, } \\
& V_{n+r, k}^{(n)}=\left(B_{n+r-1}-\frac{A_{n+r-1} B_{k}}{A_{k}}\right) V_{n+r-1, k}^{(n)}-C_{n+r-1} V_{n+r-2, k}^{(n)} \\
& +\frac{A_{n+r-1} h_{k+1}}{A_{k} h_{k}} V_{n+r-1, k+1}^{(n)}+\frac{A_{n+r-1} h_{k-1} C_{k}}{A_{k} h_{k}} V_{n+r-1, k-1}^{(n)} \text {, } \\
& V_{n+r . k}^{(n)}=0 \text { if } k \leqq n-r+1, k \neq n \text { if } r=0, V_{n, n}^{(n)}=1 \text {, } \\
& V_{n+r, n-r+2}^{(n)}=-\frac{A_{n+1} A_{n+2} \cdots A_{n+r-1} h_{n+1}}{A_{n-r+2} A_{n-r+3} \cdots A_{n} h_{n-r+2}}, r \geqq 2 .
\end{aligned}
$$

REMARK. Equation (20) holds for $k=0$ provided we set $V_{n+r-1,-1}^{(n)}=0$.

Proof. The first two items are trivial and (19) follows from (15) and (3) with $n$ replaced by $n+1$. Equation (20) is a generalization of (19) and is proved as follows. In (15), put $j=n+r$ and for $q_{n+r}\left(x_{\alpha}\right)$ use (3) with $n$ replaced by $n+r-1$. Thus with the aid of (15) we get 


$$
\begin{aligned}
V_{n+r, k}^{(n)}= & B_{n+r-1} V_{n+r-1, k}^{(n)}-C_{n+r-1} V_{n+r-2, k}^{(n)} \\
& +\frac{A_{n} A_{n+r-1} h_{n}}{h_{k}} \sum_{\alpha=0}^{n} \frac{q_{n+r-1}\left(x_{\alpha}\right) x_{\alpha} q_{k}\left(x_{\alpha}\right)}{q_{n+1}^{\prime}\left(x_{\alpha}\right) q_{n}\left(x_{\alpha}\right)} .
\end{aligned}
$$

Next, in the latter sum, for $x_{\alpha} q_{k}\left(x_{\alpha}\right)$ use (3) with $k$ instead of $n$. The stated result readily follows again with the aid of (15). Equations (21) and (22) are easily proved by induction and we omit details.

COROLLARY 2. If in the definition of orthogonality (2), $a=-b$ and $q_{n}(x)$ is even or odd according as $n$ is even or odd, then

$$
\begin{aligned}
& V_{j, k}^{(n)}=0 \text { if } j+k \text { is odd, all } n, \\
& V_{j, k}^{(n)}=\frac{2 A_{n} h_{n}}{h_{k}} \sum_{\alpha=0}^{[n / 2]} \frac{q_{j}\left(x_{\alpha}\right) q_{k}\left(x_{\alpha}\right)}{q_{n+1}^{\prime}\left(x_{x}\right) q_{n}\left(x_{\alpha}\right)}
\end{aligned}
$$

if $j+k$ is even and $n=2 r-1$ is odd,

$$
V_{j, k}=\frac{2 A_{n} h_{n}}{h_{k}} \sum_{\alpha=0}^{n / 2} \frac{q_{j}\left(x_{\alpha}\right) q_{k}\left(x_{\alpha}\right)}{q_{n+1}^{\prime}\left(x_{\alpha}\right) q_{n}\left(x_{\alpha}\right)}+\frac{A_{n} h_{n} q_{j}(0) q_{k}(0)}{h_{k} q_{n+1}^{\prime}(0) q_{n}(0)}
$$

if both $j+k$ and $n$ are even.

REMARK. The hypotheses of this corollary with $a=\infty$ and $a=1$ are satisfied $b y$ the Hermite polynomials $H_{n}(x)$ and the Jacobi polynomials $P_{n}^{(\alpha, \alpha)}(x)$ respectively. Except for normalization factors, the latter are also known as ultraspherical or Gegenbauer polynomials and include as special cases, Legendre polynomials and both kinds of Chebyshev polynomials.

Proof. Clearly $q_{n}(x) q_{n+1}^{\prime}(x)$ is even in $x$ and never vanishes for $x=x_{\alpha}$. If $n+1$ is even, we can designate the zeros of $q_{n+1}(x)$ as $\pm \alpha_{0}, \pm \alpha_{1} \cdots, \pm \alpha_{r-1}$, where $n=2 r-1$. So from (15)

$$
V_{j, k}^{(2 r-1)}=\frac{A_{n} h_{n}}{h_{k}} \sum_{\alpha=0}^{r-1} \frac{\left[1+(-)^{j+k}\right] q_{j}\left(x_{\alpha}\right) q_{k}\left(x_{\alpha}\right)}{q_{n+1}^{\prime}\left(x_{\alpha}\right) q_{n}\left(x_{\alpha}\right)}
$$

whence (23) and (24) are at hand. The proof of (25) is quite similar and we skip the details.

\section{The Gaussian quadrature formula}

It is an easy matter to recover the Gauss quadrature formula. For from (11), (13), (14) and (2), we have

$$
\begin{aligned}
\int_{a}^{b} w(x) f(x) d x & =A_{n} h_{n} \sum_{\alpha=0}^{n} \frac{f\left(x_{\alpha}\right)}{q_{n+1}^{\prime}\left(x_{\alpha}\right) q_{n}\left(x_{\alpha}\right)}+S_{2 n+2}, \\
S_{2 n+2} & =\int_{a}^{b} w(x) R_{n+1}(x) d x .
\end{aligned}
$$


The sum portion of (26) is readily recognized as the approximate Gaussian quadrature formula for the integral and $S_{2 n+2}$ is the remainder. This formula is usually proved in a different manner. See, for example, Krylov (1963), Davis (1963), and Szegö (1959). If we presume that (26) is known, the orthogonality relation (16) readily follows when we put $f(x)=q_{j}(x) q_{k}(x)$ in (26), $j, k \leqq n$. This is the proof as given in Szegö (1969). Our theorem is posed as a problem in Davis (1963), p. 360 , problem 3, and no doubt the intended proof is along the lines just noted. Our presentation seems quite natural especially in view of the results in the following section.

\section{The coefficients in the interpolation formulas}

If the expansion (8) is truncated after $n+1$ terms, we get a polynomial approximation to $f(x)$ of degree $n$. Equation (13) affords a similar approximation. In practice evaluation of the $c_{k}$ 's, see (9), might be troublesome as closed form expressions are not usually known. In numerous cases of practical interest, even when the $c_{k}$ 's can be defined in closed form, they are quite often more simply evaluated by means of recurrence relations used in the backward direction. In this connection, see Luke (1969). An alternative procedure is to evaluate $c_{k}$ by numerical integration. Indeed, if $c_{k}$ is approximated by the Gauss formula (26), (there replace $f(x)$ by $f(x) q_{k}(x)$ ), we see that $d_{k, n}$, equation (14), is an approximation for $c_{k}$. In this section we establish a connection between $c_{k}$ and $d_{k, n}$ and then obtain a useful representation for $R_{n+1}(x)$ as defined by (11).

In (14) replace $f\left(x_{\alpha}\right)$ by (8) with $x_{\alpha}$ instead of $x$. Then after a straightforward computation and use of (15) and (21), we arrive at

$$
d_{k, n}=c_{k}+\sum_{s=0}^{\infty} c_{2 n+2+s-k} V_{2 n+2+s-k, k}^{(n)},
$$

where the coefficient of $c_{2 n+2-k}$ can be deduced from (22). Equation (27) can be further simplified if (23-25) pertain. The special cases of (27) as well as (43) and (44) below corresponding to the situations where the orthogonal polynomials are those of Chebyshev have been delineated in Luke (1969), Chapter 8.

Form (8), (11), (14) and (27), we have

$$
\begin{aligned}
R_{n+1}(x) & =f(x)-f_{n}(x)=\sum_{k=0}^{\infty} c_{k} q_{k}(x)-\sum_{k=0}^{n} d_{k, n} q_{k}(x) \\
& =\sum_{k=0}^{n}\left(c_{k}-d_{k, n}\right) q_{k}(x)+\sum_{k=n+1}^{\infty} c_{k} q_{k}(x) \\
& =-\sum_{k=0}^{n} q_{k}(x) \sum_{s=0}^{\infty} c_{2 n+2+s-k} V_{2 n+2+s-k, k}^{(n)}+\sum_{k=n+1}^{\infty} c_{k} q_{k}(x),
\end{aligned}
$$

and with the aid of (18)-(22), we find 


$$
\begin{gathered}
R_{n+1}(x)=c_{n+1} q_{n+1}(x)+c_{n+2}\left[q_{n+2}(x)+C_{n+1} q_{n}(x)\right] \\
+c_{n+3}\left[q_{n+3}(x)+\frac{A_{n+2} A_{n+1} h_{n+1}}{A_{n} A_{n-1} h_{n-1}} q_{n-1}(x)-C_{n+1}\left\{\frac{A_{n+2} B_{n}}{A_{n}}-B_{n+2}\right\} q_{n}(x)\right]
\end{gathered}
$$

or

$$
R_{n+1}(x)=c_{n+1} q_{n+1}(x)\left[1+\frac{c_{n+2}}{c_{n+1}}\left(A_{n+1} x+B_{n+1}\right)+\cdots\right]
$$

in view of (3). Indeed, in (29), the coefficient of each $c_{n+r}, r \geqq 1$, must contain $q_{n+1}(x)$ as a factor since $R_{n+1}(x)=0$ when $x=x_{\alpha}, \alpha=0,1, \cdots, n$. In practice (29) and (30) can be advantageous, for even though the $c_{k}$ 's may be difficult to evaluate, asymptotic estimates are often available and these can be employed to apprise the remainder. For this and related considerations, see Luke (1969), Chapter 8 and the references given there.

For an application of the above, suppose we want to approximate $\int_{a}^{b} W(x) f(x) d x$ by $\int_{a}^{b} W(x) f_{n}(x) d x$ where $W(x)$ is not necessarily the weight function associated with the sequence of orthogonal polynomials $\left\{q_{n}(x)\right\}$. In this situation, the error is given by $\int_{a}^{b} W(x) R_{n+1}(x) d x$ where $R_{n+1}(x)$ is given by (29). In particular, if $q_{n}(x)$ is the Chebyshev polynomial of the first or second kind and $W(x)=1$, our results are applicable to the quadrature schemes discussed by Clenshaw and Curtis (1960), Elliott (1965), Fraser and Wilson (1966), Chawla (1968), Nicholson, Rabinowitz, Richter, and Zeilberger (1971), and Riess and Johnson (1972). Our error analysis differs from those propounded in these papers. Further investigation on this point is reserved for a future paper.

If in the above consideration, $W(x)=w(x)$, the weight function associated with the sequence of orthogonal polynomials $\left\{q_{n}(x)\right\}$, we then get the error for the Gauss quadrature scheme. This aspect of the subject is taken up in the next section.

\section{Error in the Gaussian quadrature formula}

Now from (26), (11), (8) and (13), (2) and (27), and because of uniform convergence of $(8)$, we have

$$
\begin{aligned}
S_{2 n+2}= & \int_{a}^{b} w(x)\left[f(x)-f_{n}(x)\right] d x=\sum_{k=0}^{\infty} c_{k} \int_{a}^{b} w(x) q_{k}(x) d x \\
& -\sum_{k=0}^{n} d_{k, n} \int_{a}^{b} w(x) q_{k}(x) d x=\frac{h_{0}}{a_{0,0}}\left(c_{0}-d_{0, n}\right),
\end{aligned}
$$

whence

$$
S_{2 n+2}=\sum_{s=0}^{\infty} c_{2 n+2+s} g_{2 n+2+s}
$$




$$
g_{2 n+2+s}=-\frac{h_{0}}{a_{0,0}} V_{2 n+2+s, 0}^{(n)}
$$

From (22),

$$
g_{2 n+2}=\frac{A_{n+1} A_{n+2} \cdots A_{2 n+1} h_{n+1}}{A_{0} A_{1} \cdots A_{n} a_{0.0}}
$$

and we prove that

$$
\begin{gathered}
g_{2 n+3}=\frac{-h_{0}}{a_{0,0}} V_{2 n+3,0}^{(n)}, \\
V_{2 n+3,0}^{(n)}=\sum_{s=0}^{n} p_{s} \xi_{s}, \xi_{0}=1, \\
p_{s}=\left(\frac{A_{2 n+2-s} B_{s}}{A_{s}}-B_{2 n+2-s}\right) \frac{A_{n+1} A_{n+2} \cdots A_{2 n+1-s} h_{n+1}}{A_{s} A_{s+1} \cdots A_{n} h_{s}}, \\
\xi_{s}=b_{0} b_{1} \cdots b_{s-1}, s>0, b_{k}=\frac{A_{2 n+2-k} h_{k+1}}{A_{k} h_{k}}, \\
\xi_{s}=\frac{A_{2 n+2} A_{2 n+1} \cdots A_{2 n+3-s} h_{s}, s>0 .}{A_{0} A_{1} \cdots A_{s-1} h_{0}}, s>0
\end{gathered}
$$

To deduce (35), put $r=n+3-s$ and $k=s$ in (20). Use (21). We then obtain the recurrence formula

$$
V_{n-s}=p_{s}+b_{s} V_{n-s-1}, V_{n-s}=V_{2 n+3-s, s}^{(n)} .
$$

Now $V_{-1}=0$ in view of (18). So $V_{0}=p_{n}$ is known and the desired solution of the difference equation (36) is

$$
\begin{aligned}
V_{n-s} & =p_{s}+\sum_{r=1}^{n-s} p_{s+r} b_{s+r-1} b_{s+r-2} \cdots b_{s}, \\
s & =n, n-1, \cdots, 0,
\end{aligned}
$$

whence (33) follows.

To get $V_{2 n+4,0}^{(n)}$, put $r=n+4-s$ and $k=s$ in (20). Then

$$
\begin{aligned}
W_{n-s}= & \left(B_{2 n+3-s}-\frac{A_{2 n+3-s} B_{s}}{A_{s}}\right) V_{2 n+3-s, s}^{(n)}-C_{2 n+3-s} V_{2 n+2-s, s}^{(n)} \\
& +\frac{C_{s} A_{2 n+3-s} h_{s-1}}{A_{s} h_{s}} V_{2 n+3-s, s-1}^{(n)}+\frac{A_{2 n+3-s} h_{s+1}}{A_{s} h_{s}} W_{n-s-1},
\end{aligned}
$$

(38) $W_{n-s}=V_{2 n+4-s, s}^{(n)}$.

Both $V_{2 n+2-s, s}^{(n)}$ and the same with $s$ replaced by $s-1$ are known from (22). Since $W_{-1}=0, W_{0}$ is known and the wanted solution of (38) follows after the manner of that for (36). $V_{2 n+r, 0}^{(n)}, r>4$, can be developed in a similar fashion. 
The form (32) is advantageous for the study of the error since the coefficients $c_{k}$ depend only on $w(x)$ and $f(x)$ while the coefficients $g_{k}$ depend on elements associated with the system of orthogonal polynomials. Thus in the absence of closed form expressions for $g_{k}$, the early coefficients for use in (32) can be tabulated once and for all upon specification of the system of orthogonal polynomials and associated weight function.

Under fairly general conditions, it is known that expansions in series of orthogonal functions converge in the mean to the functions associated with them. But for most purposes in applied mathematics, mean convergence is not strong enough. We usually require at least point wise convergence. Indeed, in our present analysis we require uniform convergence to deduce (32). Unfortunately, except for expansions in series of Jacobi polynomials, very little is known on pointwise convergence. If $f(x)$ is analytic in the closed interval $-1 \leqq x \leqq 1$, then its associated Jacobi series is convergent in the interior of the largest ellipse with foci at \pm 1 in which $f(x)$ is analytic. If $f(x)$ is analytic as above and we expand in Legendre or Chebyshev polynomials, then we have uniform convergence. However, the form for $S_{2 n+2}$ might well be suitable for other systems of orthogonal polynomials when $f(x)$ is analytic. Further, it might also be applicable when $f(x)$ is not analytic on the path of integration. In the absence of theoretical criteria, it appears that we must rely on heuristic means for guidance on these and other related questions. In this connection, we also recognize that only the lead term of (32) is known in closed form. The higher order terms can be determined by summation and in particular cases, see the next section, explicit expressions for some of the higher order terms are found. In view of the above remarks, our analysis is not complete. Some exploratory numerical work is underway, but we postpone further considerations for a future paper.

In some recent work*, Chawla (1971) takes up the special case when $f(x)$ is analytic and is expanded in series of Legendre polynomials. He shows that for $s$ fixed and $n$ large,

$$
g_{2 n+2+2 s}=(\pi / 2 n)^{\frac{1}{2}} \frac{(2 n+3)(2 s-2) !}{2^{2 s}(2 n+3+s)(2 s-1)(s !)^{2}}\left[1+0\left(n^{-1}\right)\right] .
$$

For $s=0$, he obtains $g_{2 n+2}$ precisely as do we, see (44) with $\alpha=\beta=0$. Actually, Chawla writes $g_{2 n+2+2 s} \simeq$ etc. without $O\left(n^{-1}\right)$ as above. In any event, it is important to realize that $O\left(n^{-1}\right)$ is $s$ dependent. This aside he writes

$$
S_{2 n+2} \simeq(\pi / 2 n)^{\frac{1}{2}}\left(c_{2 n+2}-\frac{1}{2} c_{2 n+4}-\frac{1}{8} c_{2 n+6} \cdots\right) .
$$

If the coefficients $c_{k}^{*}$ are known in

$$
f(x)=\sum_{k=0}^{\infty} c_{k}^{*} T_{k}(x)
$$

* I am grateful to the referee for pointing out this reference. 
where $T_{k}(x)$ is the Chebyshev polynomial of the first kind, Chawla shows that for Gauss-Legendre quadrature

$$
S_{2 n+2} \simeq \frac{\pi}{2}\left(c_{2 n+2}^{*}-c_{2 n+4}^{*}\right) .
$$

For the example $f(x)=\left(9 x^{2}+1\right)^{-1}$, the latter estimate is in good agreement with the true error even though $f(x)$ has singularities near the path of integration. For an integrable singularity on the path of integration, e.g. $f(x)=(x+1)^{\frac{1}{2}}$, the referee states that in some unpublished work $c_{2 n+2} g_{2 n+2}$ is a poor approximation to the error. Thus several interesting questions arise in connection with the use of (32), especially for functions which are not analytic. But, as previously remarked, we defer further comments for a future paper.

\section{Evaluation of error coefficients in particular cases}

It is of interest to compute $g_{2 n+2}$ and $g_{2 n+3}$ in four cases of practical interest. In one case, we also get $g_{2 n+4}$. We follow the notation in Luke (1969), Chapter 8 and Erdelyi et al. (1953).

CASE 1. Jacobi Polynomials

$$
\begin{aligned}
a & =-1, b=1, w(x)=(1-x)^{\alpha}(1+x)^{\beta}, \\
q_{n}(x)=P_{n}^{(\alpha, \beta)}(x) & =\frac{(\alpha+1)_{n}}{n !}{ }_{2} F_{1}\left(\begin{array}{c}
-n, n+\lambda \\
\alpha+1
\end{array} \mid \frac{1-x}{2}\right), \lambda=\alpha+\beta+1, \\
h_{n} & =\frac{2^{\lambda} \Gamma(n+\alpha+1) \Gamma(n+\beta+1)}{(2 n+\lambda) n ! \Gamma(n+\lambda)},
\end{aligned}
$$

$$
A_{n}=\frac{(2 n+\lambda)(2 n+\lambda+1)}{2(n+1)(n+\lambda)}, \quad B_{n}=\frac{\left(\alpha^{2}-\beta^{2}\right)(2 n+\lambda)}{2(n+1)(n+\lambda)(2 n+\lambda-1)} .
$$

Then

$$
\begin{aligned}
g_{2 n+2} & =\frac{2^{\lambda} \Gamma(n+\alpha+2) \Gamma(n+\beta+2) \Gamma(n+\lambda+1)(n+1) ! \Gamma(4 n+\lambda+4)}{[\Gamma(2 n+\lambda+2)]^{2} \Gamma(2 n+\lambda+3)(2 n+2) !} \\
& =(\pi / 2 n)^{\frac{1}{2}}\left[1+0\left(n^{-1}\right)\right] .
\end{aligned}
$$

Also

$$
\begin{aligned}
& p_{s} \xi_{s}=\frac{\left(\alpha^{2}-\beta^{2}\right) \Gamma(\lambda+1) \Gamma(n+\alpha+2) \Gamma(n+\beta+2) \Gamma(n+\lambda+1)(n+1) ! \Gamma(4 n+\lambda+6)}{2 \Gamma(\alpha+1) \Gamma(\beta+1) \Gamma(2 n+\lambda+2)[\Gamma(2 n+\lambda+3)]^{2}(2 n+3) !} \\
& \text { (45) } \times\left[\frac{1}{(2 s+\lambda+1)(2 s+\lambda-1)}-\frac{1}{(4 n+5+\lambda-2 s)(4 n+3+\lambda-2 s)}\right] .
\end{aligned}
$$

Now 


$$
\sum_{s=0}^{n}[(2 s+\lambda+1)(2 s+\lambda-1)]^{-1}=\frac{(n+1)}{(\lambda-1)(2 n+\lambda+1)},
$$

(46) $\sum_{s=0}^{n}[(4 n+\lambda+5-2 s)(4 n+\lambda+3-2 s)]^{-1}=\frac{(n+1)}{(2 n+\lambda+3)(4 n+\lambda+5)}$.

Thus after some algebra. we get

$g_{2 n+3}=-\frac{2^{\lambda+1}(\alpha-\beta)(n+1) \Gamma(n+\alpha+2) \Gamma(n+\beta+2) \Gamma(n+\lambda+1)(n+2) ! \Gamma(4 n+\lambda+5)}{(2 n+\lambda+1)[\Gamma(2 n+\lambda+2)]^{2} \Gamma(2 n+\lambda+4)(2 n+3) !}$,

$$
g_{2 n+3}=-(\alpha-\beta)(\pi / 2 n)^{\frac{1}{2}}\left[1+0\left(n^{-1}\right)\right] .
$$

If $\alpha=\beta, g_{2 n+3}=0$ which is in agreement with (23). In a similar fashion we get the following data for $g_{2 n+4}$.

$$
g_{2 n+4}=E_{1} F_{1}+E_{2} F_{2}+E_{3} F_{3},
$$

$$
\begin{aligned}
E_{1} & =-\frac{\left(\alpha^{2}-\beta^{2}\right)^{2} \theta}{(2 n+\lambda+1)(2 n+\lambda+3)}, \quad E_{2}=-\frac{\theta}{2 n+\lambda+2}, \quad E_{3}=-E_{2}, \\
\theta & =\frac{2^{\lambda}(n+1) ! \Gamma(n+\alpha+2) \Gamma(n+\beta+2) \Gamma(n+\lambda+1) \Gamma(4 n+\lambda+8)}{(2 n+4) !\left[(\Gamma(2 n+\lambda+2)]^{2} \Gamma(2 n+\lambda+4)\right.} \\
(49) & =16(2 \pi n)^{\frac{1}{2}}\left[1+0\left(n^{-1}\right)\right], \\
F_{1} & =G_{1}-G_{2}, G_{1}=\sum_{k=0}^{n} a_{k}, G_{2}=\sum_{k=0}^{n} b_{k}, \\
a_{k} & =\frac{(n+1-k)(n+2-k)}{(4 n+5-2 k+\lambda)^{2}(4 n+7-2 k+\lambda)(2 k+\lambda-1)}, \\
b_{k} & =\frac{(n+1-k)(n+2-k)}{(4 n+5-2 k+\lambda)(2 k+\lambda-1)^{2}(2 k+\lambda+1)},
\end{aligned}
$$

(50) $a_{k}-b_{k}=-\frac{4(n+1-k)(n+2-k)(2 n+3-2 k)(2 n+3+\lambda)}{(4 n+5-2 k+\lambda)^{2}(4 n+7-2 k+\lambda)(2 k+\lambda-1)^{2}(2 k+\lambda+1)}$,

$$
F_{2}=\sum_{k=0}^{n} \frac{(2 n+3-k)(2 n+3-k+\alpha)(2 n+3-k+\beta)(2 n+2-k+\lambda)}{(4 n+4-2 k+\lambda)(4 n+5-2 k+\lambda)^{2}(4 n+6-2 k+\lambda)}
$$

$$
\begin{aligned}
& =\frac{(n+1)}{16}\left[1+0\left(n^{-1}\right)\right] \\
& F_{3}=\sum_{k=1}^{n} \frac{k(k+\alpha)(k+\beta)(k+\lambda-1)}{(2 k+\lambda-2)(2 k+\lambda-1)^{2}(2 k+\lambda)} \\
& =\frac{(n+1)}{16}\left[1+0\left(n^{-1}\right)\right] .
\end{aligned}
$$


In certain special cases, the required sums in (50), (51) and (52) can be expressed in closed form. Here care must be exercised in that $k$ is a discrete parameter and must first be assigned a value before assigning values to $\alpha$ and $\beta$.

CASE 1. $\alpha=\beta, \lambda=2 \alpha+1$.

(53) $E_{1} F_{1}=0, E_{2} F_{2}=\frac{\theta(n+1)}{16(2 n+2 \alpha+3)}\left[1+\frac{\left(\frac{1}{4}-\alpha^{2}\right)}{\left(n+\alpha+\frac{5}{2}\right)\left(2 n+\alpha+\frac{7}{2}\right)}\right]$,

$$
E_{3} F_{3}=-\frac{\theta n}{16(2 n+2 \alpha+3)}\left[1+\frac{\left(\frac{1}{2}-\alpha\right)}{n+\alpha+\frac{1}{2}}\right] \text { if } \alpha \neq-\frac{1}{2} \text {, }
$$

(54) $E_{3} F_{3}=-\frac{\theta}{32}$ if $\alpha=-\frac{1}{2}$,

$$
\begin{gathered}
g_{2 n+4}=-\frac{\theta(n+1)\left(\frac{1}{2}+\alpha\right)}{16(2 n+2 \alpha+3)\left(n+\alpha+\frac{1}{2}\right)}\left[1+\frac{\left(\frac{1}{2}-\alpha\right)\left(n+\alpha+\frac{1}{2}\right)}{\left(n+\alpha+\frac{5}{2}\right)\left(2 n+\alpha+\frac{7}{2}\right)}\right] \\
g_{2 n+4}=-\left(\frac{1}{2}+\alpha\right)(\pi / 2 n)^{\frac{1}{2}}\left[1+O\left(n^{-1}\right)\right] .
\end{gathered}
$$

CASE 2. $\alpha=-\beta, \lambda=1$

$$
\begin{aligned}
E_{1} G_{1} & =0, \quad E_{1} G_{2}=\frac{\alpha^{2} \theta}{4(2 n+3)}, \\
E_{2} F_{2} & =-\frac{\theta(n+1)}{16(2 n+3)}\left[1+\frac{\left(1-4 \alpha^{2}\right)}{(2 n+5)(4 n+7)}\right], \\
E_{3} F_{3} & =\frac{\theta n}{16(2 n+3)}\left[1+\frac{\left(1-4 \alpha^{2}\right)}{2 n+1}\right], \\
g_{2 n+4}= & -\frac{\theta\left(1-4 \alpha^{2}\right)(n+1)}{16(2 n+1)(2 n+3)}\left[1+\frac{(2 n+1)}{(2 n+5)(4 n+7)}\right], \\
g_{2 n+4}=-\frac{1}{2}\left(1-4 \alpha^{2}\right)(\pi / 2 n)^{4} & {\left[1+0\left(n^{-1}\right)\right] . }
\end{aligned}
$$

CASE II. Generalized Laguerre Polynomials.

$$
\begin{gathered}
a=0, \quad b=\infty, w(x)=e^{-x} x^{\alpha} \\
q_{n}(x)=L_{n}^{(\alpha)}(x)=\lim _{\beta \rightarrow \infty} P_{n}^{(\alpha, \beta)}(1-2 x / \beta)=\frac{(\alpha+1)_{n}}{n !}{ }_{1} F_{1}(-n ; \alpha+1 ; x), \\
h_{n}=\frac{\Gamma(n+\alpha+1)}{n !}, \quad A_{n}=-\frac{1}{n+1}, \quad B_{n}=\frac{2 n+\alpha+1}{n+1} .
\end{gathered}
$$

We find

$$
g_{2 n+2}=\frac{\Gamma(n+\alpha+2)(n+1) !}{(2 n+2) !}=\frac{\pi^{\frac{1}{2}} n^{\alpha+\frac{1}{2}}}{2^{2 n+2}}\left[1+0\left(n^{-1}\right)\right],
$$




$$
\begin{aligned}
g_{2 n+3} & =\frac{2 \Gamma(n+\alpha+2)(n+2) !(n+1)}{(2 n+3) !}=\frac{\pi^{\frac{3}{3}} n^{\alpha+1}}{2^{2 n+2}}\left[1+0\left(n^{-1}\right)\right], \\
g_{2 n+4} & =\frac{(n+1) ! \Gamma(n+\alpha+2)(n+1)}{6(2 n+4) !}(n+3)[2(n+1)(n+2)+1-\alpha] \\
& =\frac{\pi^{\frac{1}{4} n^{\alpha+1}}}{2^{2 n+3}}\left[1+0\left(n^{-1}\right)\right] .
\end{aligned}
$$

Case III. Hermite Polynomials

$$
\begin{aligned}
a= & -\infty, \quad b=\infty, \quad w(x)=e^{-x^{2}}, \\
q_{n}(x)= & H_{n}(x), \quad H_{2 m+\varepsilon}(x)=(-)^{m} 2^{2 m+\varepsilon} m ! x^{\varepsilon} L_{m}^{\left(\varepsilon-\frac{1}{2}\right)}\left(x^{2}\right), \\
& \varepsilon=0 \quad \text { or } \varepsilon=1, \\
h_{n}= & \pi^{\frac{1}{2}} 2^{n} n !, \quad A_{n}=2, \quad B_{n}=0 .
\end{aligned}
$$

Then

$$
\begin{aligned}
g_{2 n+2} & =h_{n+1}=\pi^{\frac{1}{2}} 2^{n+1}(n+1) ! \\
g_{2 n+4} & =-\pi^{\frac{1}{2}} 2^{n+2}(n+1) !(n+1)(n+3), \\
g_{2 n+2 r+1} & =0, \quad r=0,1, \cdots .
\end{aligned}
$$

CASE IV. Bessel Polynomials

Though the basic theory has been developed for real polynomials over real paths, it is obvious that the results hold for complex polynomials over complex paths. The Bessel polynomials are useful for the numerical inversion of Laplace transforms, see [9, Vol. 2, pp. 194, 253]. We have

$$
\begin{gathered}
(2 \pi i)^{-1} \int_{C} \frac{e^{z} Q_{n}(v,-z) Q_{m}(v,-z)}{z^{v+1}} d z=\frac{(-)^{n} n ! \delta m n}{(2 n+v) \Gamma(n+v)}, \\
Q_{n}(v,-z)={ }_{2} F_{0}\left(-n, n+v ; \frac{1}{z}\right),
\end{gathered}
$$

where $C$ is the path $c-i \infty$ to $c+i \infty, c>0$. Also

$$
A_{n}=-\frac{(2 n+v)(2 n+v+1)}{n+v}, \quad B_{n}=\frac{(v-1)(2 n+v)}{(n+v)(2 n+v-1)} .
$$

Then

(70) $g_{2 n+2}=\frac{(-)^{n+1} \Gamma(n+v+1)(n+1) ! \Gamma(4 n+v+4)}{[\Gamma(2 n+v+2)]^{2} \Gamma(2 n+v+3)}$

$$
=\frac{(-)^{n+1} 2^{2 n+(3 / 2)-v}}{n^{v}}\left[1+0\left(n^{-1}\right)\right] \text {, }
$$


(71) $g_{2 n+3}=\frac{(-)^{n} 4(n+1) \Gamma(n+v+1)(n+2) ! \Gamma(4 n+v+5)}{(2 n+v+1)[\Gamma(2 n+v+2)]^{2} \Gamma(2 n+v+4)}$

$$
=\frac{(-)^{n} 2^{2 n+(7 / 2)-1}}{n^{n-1}}\left[1+0\left(n^{-1}\right)\right]
$$

(72)

$$
g_{2 n+4}=E_{1} F_{1}+E_{2} F_{2}+E_{3} F_{3},
$$

$$
E_{1}=\frac{4(v-1)^{2} \phi}{(2 n+v+1)(2 n+v+3)}, \quad E_{2}=-\frac{\phi}{2 n+v+2}, \quad E_{3}=-E_{2},
$$

(73) $\phi=(-)^{n} \frac{(n+1) ! \Gamma(n+v+1) \Gamma(4 n+v+8)}{[\Gamma(2 n+v+2)]^{2} \Gamma(2 n+v+4)}$

$$
=(-)^{n} n^{3-v} 2^{2 n-v+17 / 2}\left[1+O\left(n^{-1}\right)\right] \text {, }
$$

(74) $F_{1}=G_{1}-G_{2}$,

where $G_{1}$ and $G_{2}$ are given by (50) with $\lambda$ replaced by $v$,

$$
\begin{aligned}
F_{2} & =\sum_{k=0}^{n} \frac{(2 n+3-k)(2 n+2-k+v)}{(4 n+4+2 k+v)(4 n+5-7 k+v)^{2}(4 n+6-2 k+v)} \\
F_{3} & =\sum_{k=1}^{n} \frac{k(k+v-1)}{(2 k+v-2)(2 k+v-1)^{2}(2 k+v)} . \\
\text { If } v & =1,
\end{aligned}
$$

$$
g_{2 n+4}=-\frac{\phi(n+1)}{4(2 n+1)(2 n+3)}\left[1+\frac{2 n+1}{(2 n+5)(4 n+7)}\right] .
$$

\section{References}

M. M. Chawla (1968), 'Frror Fstimates for the Clenshaw-Curtis Quadrature', Math. Comp. 22, 65I-656.

M. M. Chawla (1971), Asymptotic Gauss Quadrature Errors as Fourier Coefficients of the Integrand', J. Austral. Math. Soc. 12, 315-322.

C. W. Clenshaw, and A. R. Curtis (1960), 'A Method for Numerical Integration on an.Automatic, Computer', Numer. Math. 2, 197-205.

P. J. Davis (1963), Interpolation and Approximation, (Blaisdell, New York, 1963).

D. Elliott (1965), 'Truncation Errors in Two Chebyshev Series Expansions', Math. Comp. 19. 234-248.

A. Erdelyi (1953), et al, Higher Transcendental Functions, Vol. 2, (McGraw-Hill, New York. 1953).

W. Fraser, and M. M. Wilson (1966), 'Remarks on the Clenshaw-Curtis Quadrature Scheme', SIAM Rev. 8, 322-327. 
V. I. Krylov (1962), Approximate Calculation of Integrals, (translated by A. H. Stroud), (MacMillan, New York, 1962).

Y. L. Luke (1969), The Special Functions and Their Approximations, Vols. 1, 2, (Academic Press, New York, 1969).

D. Nicholson, P. Rabinowitz, N. Richter and D. Zeilberger (1971), 'On the Error in the Numerical Integration of Chebyshev Polynomials', Math. Comp. 25, 79-86.

R. D. Riess and L. W. Johnson (1972), 'Error Estimates for Clenshaw-Curtis Quadrature', Numer. Math. 18, 345-353.

G. Szego, Orthogonal Polynomials (1959). (Colloq. Publ., Vol. 23). (Amer. Math. Soc., Providence, R. I., 1959).

Department of Mathematics

University of Missouri

Kansas City, Missouri, 64110

U.S.A. 